POS PROCEEDINGS

\title{
Design of Beam Halo Monitor for CMS
}

\author{
Styliani Orfanelli ${ }^{1}$ \\ CERN \\ Geneva, Switzerland \\ National Technical University of Athens \\ Athens, Greece \\ E-mail: styliani.orfanelliecern.ch
}

\author{
Anne Dabrowski, Rob Loos \\ CERN \\ Geneva, Switzerland \\ E-mail: anne.evelyn.dabrowskilcern.ch, robert.loos@cern.ch
}

Fabrizio Fabbri, Alessandro Montanari, Nicolo Tosi ${ }^{2}$

INFN

Bologna, Italy

E-mail: fabrizio.fabbriabo.infn.it, montanariabo.infn.it, nicolo.tosiecern.ch

\author{
Alexey Finkel, Roger Rusack \\ University of Minnesota \\ Minnesota, USA \\ E-mail: alexey.finkelecern.ch, rusackephysics.umn.edu
}

\section{David P. Stickland}

Princeton University

Princeton, USA

E-mail: david.sticklan@cern.ch

1

Speaker

Also at University of Bologna, Bologna, Italy 
A fast and directional monitoring system for the CMS experiment is designed to provide an online, bunch-by-bunch measurement of beam background induced by beam halo interactions, separately for each beam. The background detection is based on Cherenkov radiation produced in synthetic fused silica read out by a fast, UV sensitive photomultiplier tube. Twenty detector units per end will be azimuthally distributed around the rotating shielding of CMS, covering $\sim 408 \mathrm{~cm}^{2}$ at $20.6 \mathrm{~m}$ from the interaction point, at a radius of $\sim 180 \mathrm{~cm}$. The directional and fast response of the system allows the discrimination of the background particles from the dominant flux in the cavern induced by pp collision debris, produced within the $25 \mathrm{~ns}$ bunch spacing. A robust multi-layered shielding will enclose each detector unit to protect the photomultiplier tube from the magnetic field and to eliminate the occupancy from low energy particles. The design of the front-end units is validated by experimental results. An overview of the new system to be integrated in CMS during the current shutdown of LHC will be presented, and its perspective for monitoring in High Luminosity LHC.

Technology and Instrumentation in Particle Physics 2014

2-6 June, 2014

Amsterdam, the Netherlands 


\section{Introduction}

Monitoring of beam conditions is essential for high quality physics data-taking in CMS experiment. In 2015 the LHC will operate with as higher beam intensity, tighter collimator settings and exceeding nominal luminosity, which shall increase the potential of the Machine Induced Background (MIB) from LHC, i.e. products of secondary cascades, initiated by proton losses around LHC. A novel Beam Halo Monitoring system has been designed to provide an online bunch-by-bunch measurement of MIB arriving CMS at high radius $(\sim 1.8 \mathrm{~m}$ from beam axis), separately for the two beams. The system is an array of fast, directional Cherenkov detectors that will be distributed azimuthally outside the rotating shielding of CMS, $20.625 \mathrm{~m}$ from the interaction point (IP), and will flag at real-time adverse beam conditions to CMS and LHC, complementing the measurement by the diamond-sensor based BCM1F system at lower radius and closer to the IP.

\section{Principles of Machine Induced Background detection}

CMS radiation environment and the need of suppression the vast flux of particles produced from pp collisions, arriving at the opposite direction of the MIB and dominating in CMS cavern, set the primary constraints of the BHM design. The key features of this system are the choice of location that allows the use of time-of-flight techniques and the prompt and directional nature of Cherenkov light.

\section{1 "Golden" timing location}

Golden locations are the locations in the CMS cavern, where the MIB would arrive with a maximum time separation from the collision products. For the $25 \mathrm{~ns}$ bunch spacing this corresponds to $12.5 \mathrm{~ns}$ (see Fig.1, left). The 6th golden location, at $20.625 \mathrm{~m}$ from CMS IP, outside the rotating shielding (see Fig. 1, right) is the most appropriate to install BHM. It is easily accessible in CMS cavern, with space availability, less ferocious environmental conditions i.e. radiation levels and magnetic field and increased ratio of MIB over the pp products flux with respect to the other golden locations.
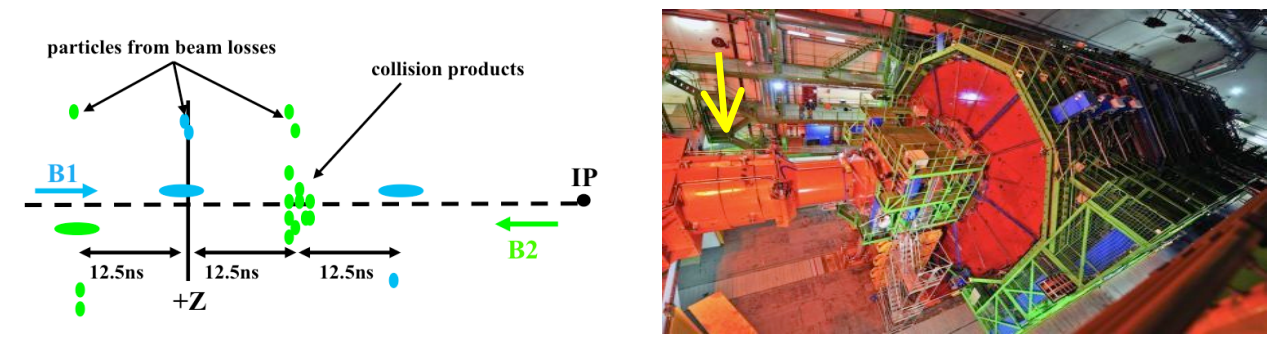

Figure 1. Time separation in Golden Locations (left). Golden location 6 (yellow arrow) (right).

\subsection{Properties of Machine Induced Background at Golden Location 6}

BHM will be mostly sensitive to beam background muons, so-called Beam Halo Muons, which are a result of proton interactions at the beam collimators. Based on FLUKA simulations 
for nominal LHC the beam halo muons arriving at the BHM location have a mean energy of 10$20 \mathrm{GeV}$, arrive parallel with the incoming beam and are concentrated in angle. The MIB flux is estimated to be $Q\left(\mathrm{~Hz} / \mathrm{cm}^{2}\right)$. The respective distribution of energy and angle of pp muons (products of pp collisions) shows that they arrive straight from the IP, in the opposite direction of MIB ( $180^{\circ}$ difference) . The pp-muon flux is three orders of magnitude larger than the MIB muon flux, meaning that the BHM unit has to suppress the signal produced by muons arriving from the CMS side and not the tunnel to $0.1 \%$.
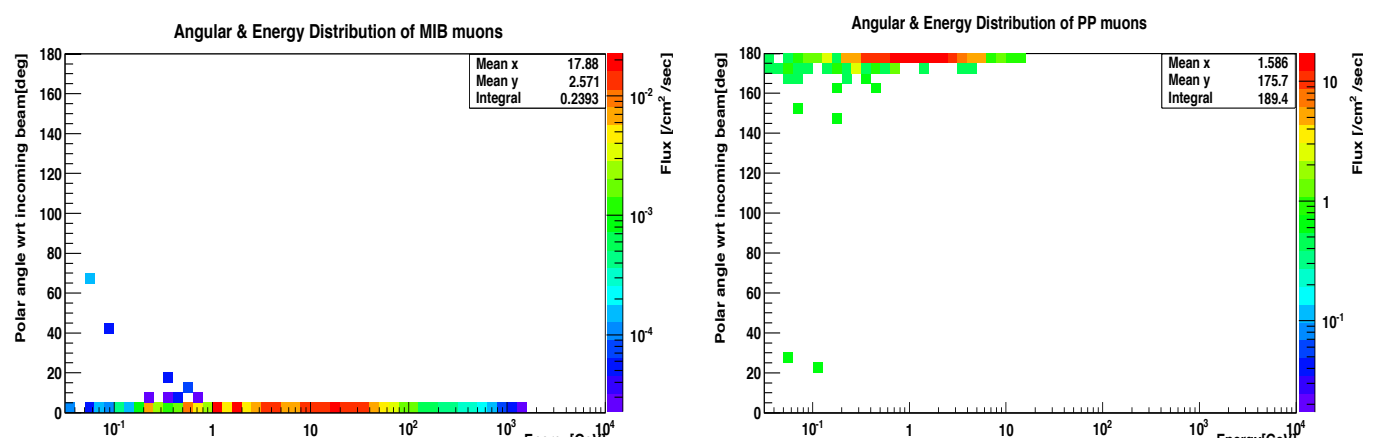

Figure 2. Energy and angular distributions of MIB (left) and pp (right) muons at Golden Location 6.

\subsection{Cherenkov radiation}

Cherenkov radiation fulfills all the requirements of BHM system and it is the technology behind this novel detector. Cherenkov light is prompt and enables the measurement within the $12.5 \mathrm{~ns}$ time window for the MIB measurement. It is directional, which allows the discrimination of the direction of the particles based on the difference on the light yield produced. What is more, it is produced only by charged particles making BHM insensitive to any neutron or photons of the CMS cavern. The radiator material is synthetically fused quartz, which is radiation hard for at least $100 \mathrm{krad}$ (Fig. 3).

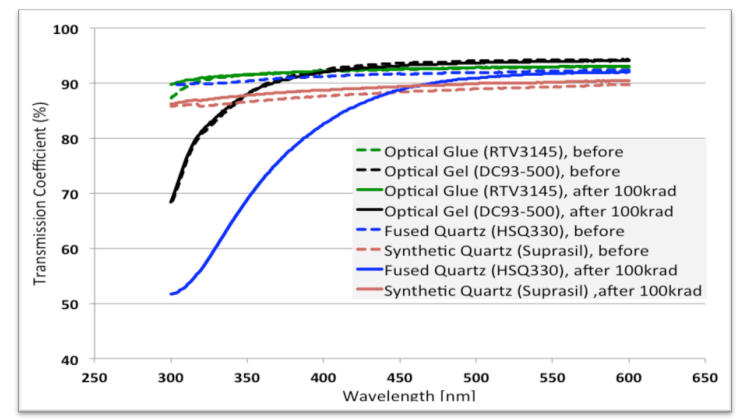

Figure 3. Transmission coefficient of silicon based material before and after irradiation of $100 \mathrm{krad}$.

\section{Detector Unit}

A BHM detector unit is shown in Figure 4: a $10 \mathrm{~cm}$ synthetically fused quartz bar blackpainted on the front side of $5.1 \mathrm{~cm}$ diameter, $1 \mathrm{~mm}$ optical coupling RTV1345, a fast photomultiplier R2059 Hamamatsu of $5.1 \mathrm{~cm}$ diameter, triple layer magnetic shielding made of $0.6 \mathrm{~mm}$ permalloy, $1 \mathrm{~mm} \mu$ metal and $1 \mathrm{~cm}$ thick ARMCO soft iron box. The iron shielding serves as a protection of the PMT from the $17 \mathrm{mT}$ residual magnetic field and from low energy e-/e+ produced by pp collisions, arriving at the same direction with the MIB. 

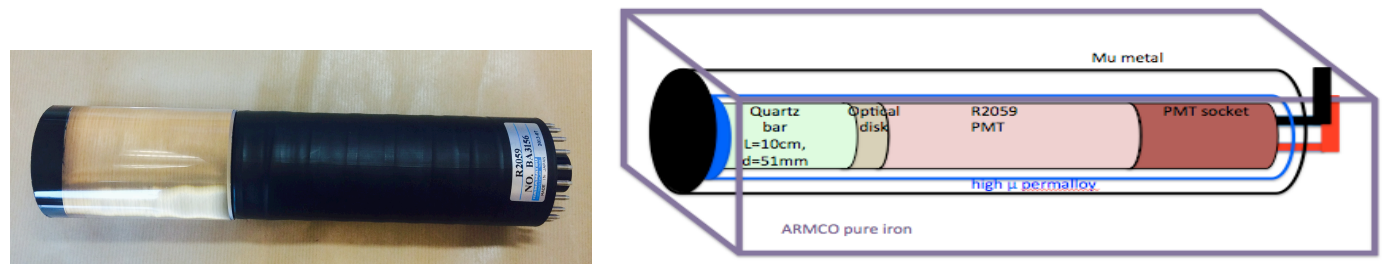

Figure 4. The quartz bar optically coupled to R2059 (left). BHM detector unit sketch (right).

\section{Directionality and Timing performance during test beams}

The performance of the timing and the directional properties of the BHM detector unit has been tested during test beams (Figure 5, left).
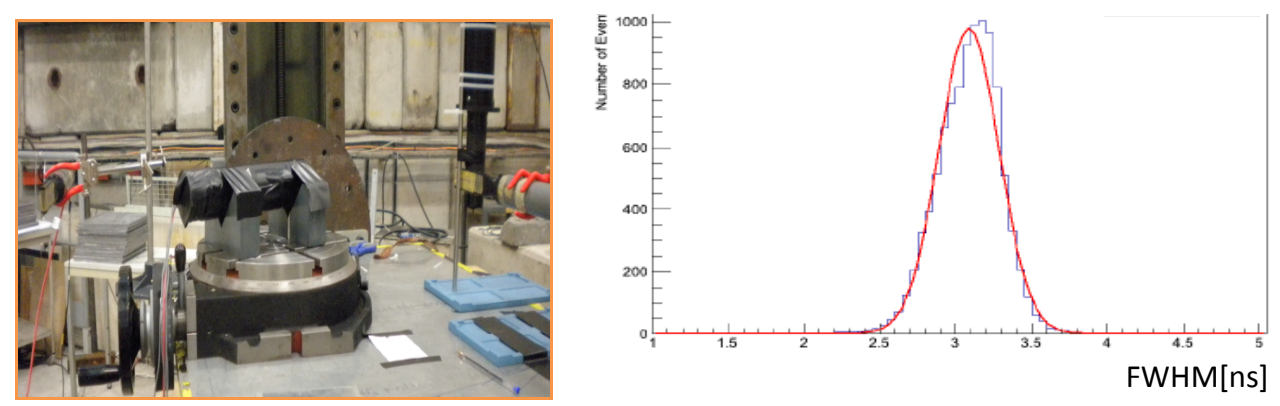

Figure 5. Test-beam setup: BHM unit stands on a rotating base, allowing the measurement of signal from particles arriving from different angles (left). FWHM 3.1ns of BHM signal during test-beam (right).

To benefit from the placement at the golden location the signal needs to be faster than 10ns. Figure 5 (right) shows the fast timing distribution of the signal during a test beam in T9 CERN July 2012 equal to $\sim 3.1 \mathrm{~ns}$, which is faster than the required $12.5 \mathrm{~ns}$.

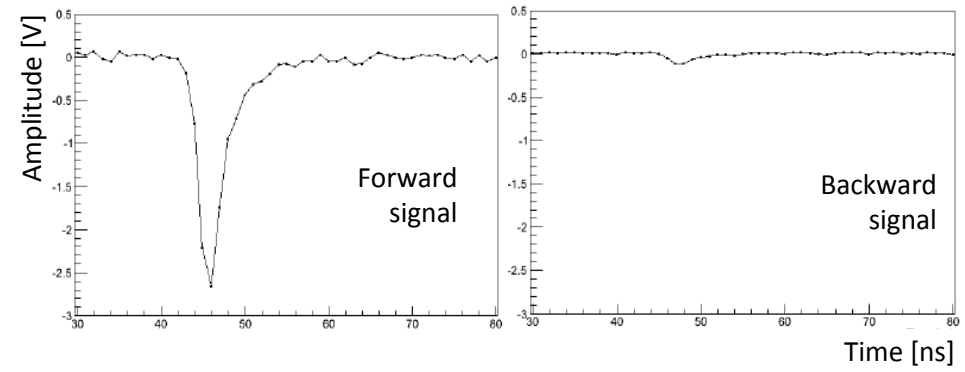

Figure 6. Signal produced from a particle arriving from the front/back side of the BHM unit (left/right).

Directionality is the property of the detector to distinguish particles coming from different directions and is used to suppress background signal while selecting particles parallel and consistent with the direction of the incoming beam. During test-beams it was validated that the suppression of the backward signal to 1 over 1000 is achievable; by setting a discrimination voltage, e.g. $4.8 \mathrm{~V}$ in fig. 5, $\sim 90 \%$ of the forward signal is accepted, while the backward signal acceptance is suppressed to $O\left(10^{-4}\right)$. 

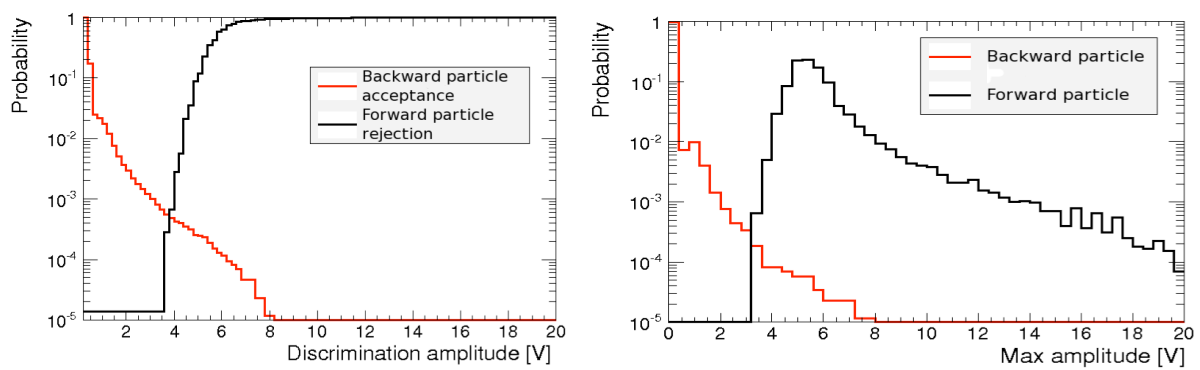

Figure 7. Normal (left) and cumulative (right) amplitude distributions from test beam data.

\section{System overview}

Each detector unit is based on $51 \mathrm{~mm}$ diameter quartz bars (cross section of $20.4 \mathrm{~cm}^{2}$ ), while the MIB rate is $O\left(\mathrm{~Hz} / \mathrm{cm}^{2}\right)$ in good LHC conditions. For a rate of $\sim 1 \mathrm{MIB}$ hit/bunch crossing, an acceptance of $\sim 400 \mathrm{~cm}^{2}$ is needed, meaning that 20 units per end are required. They will be azimuthally distributed around the rotating shielding, with overlapping acceptance with the CSC chambers and placed completely parallel to the beam axis with the front face towards the tunnel.

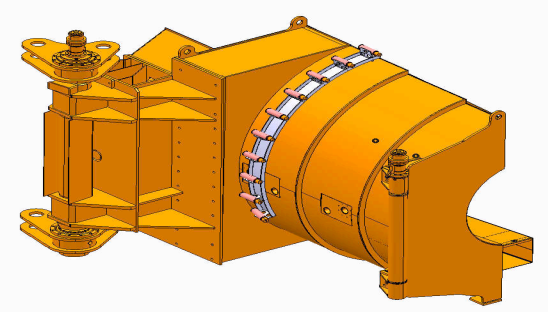

Figure 8. The azimuthal distribution of ten BHM detector units around the CMS rotating shielding.

\section{HL-LHC era}

Beam losses, being proportional to the luminosity, should result on an increase of beam halo muons creation at the collimators (five times the nominal L) for HL-LHC. Tighter collimator settings will be another reason for increased MIB. The pile up for HL-LHC is expected to be $\sim 140$ - five times more than the nominal- resulting the conservation of the MIB over pp ratio. That means that BHM will be fully operational for HL-LHC and shall not be affected by the ferocious environment of HL-LHC.

\section{Summary}

A new Cherenkov-based MIB monitoring system has been designed and will be installed during LS1. Twenty channels per end will be azimuthally distributed around CMS rotating shielding. The system has a response of $3.1 \mathrm{~ns}$ and is able to distinguish MIB from pp based on time of arrival. Suppression of the signal produced from backward particles is suppressed by directionality to $0.1 \%$. The detector unit is based on synthetically fused silica, R2059 Hamamatsu PMT and a triple layer of shielding to suppress collision induced background signal and the residual magnetic field. The system performance has been verified during test beams. The system is designed for the CMS radiation environment and will survive at that location for $>3000 \mathrm{fb}^{-1}$, being fully functional for HL-LHC. 